\title{
Human Embryonic Stem Cells Express Elevated Levels of Multiple Pro-Apoptotic BCL-2 Family Members
}

\author{
David T. Madden ${ }^{1,2 *}$, Diana Davila-Kruger ${ }^{2}$, Simon Melov², Dale E. Bredesen ${ }^{2,3}$
}

1 College of Pharmacy, Touro University - California, Vallejo, California, United States of America, 2 Buck Institute for Research on Aging, Novato, California, United States of America, 3 University of California, San Francisco, San Francisco, California, United States of America

\begin{abstract}
Two of the greatest challenges in regenerative medicine today remain (1) the ability to culture human embryonic stem cells (hESCs) at a scale sufficient to satisfy clinical demand and (2) the ability to eliminate teratoma-forming cells from preparations of cells with clinically desirable phenotypes. Understanding the pathways governing apoptosis in hESCs may provide a means to address these issues. Limiting apoptosis could aid scaling efforts, whereas triggering selective apoptosis in hESCs could eliminate unwanted teratoma-forming cells. We focus here on the BCL-2 family of proteins, which regulate mitochondrial-dependent apoptosis. We used quantitative PCR to compare the steady-state expression profile of all human BCL-2 family members in hESCs with that of human primary cells from various origins and two cancer lines. Our findings indicate that hESCs express elevated levels of the pro-apoptotic BH3-only BCL-2 family members NOXA, BIK, BIM, BMF and PUMA when compared with differentiated cells and cancer cells. However, compensatory expression of pro-survival BCL-2 family members in hESCs was not observed, suggesting a possible explanation for the elevated rates of apoptosis observed in proliferating hESC cultures, as well as a mechanism that could be exploited to limit hESC-derived neoplasms.
\end{abstract}

Citation: Madden DT, Davila-Kruger D, Melov S, Bredesen DE (2011) Human Embryonic Stem Cells Express Elevated Levels of Multiple Pro-Apoptotic BCL-2 Family Members. PLoS ONE 6(12): e28530. doi:10.1371/journal.pone.0028530

Editor: Dimas Tadeu Covas, University of Sao Paulo - USP, Brazil

Received September 30, 2011; Accepted November 9, 2011; Published December 9, 2011

Copyright: (C) 2011 Madden et al. This is an open-access article distributed under the terms of the Creative Commons Attribution License, which permits unrestricted use, distribution, and reproduction in any medium, provided the original author and source are credited.

Funding: The work was supported by a SEED grant from the California Institute of Regenerative Medicine (RS1-00163-1), as well as a postdoctoral fellowship from the Larry L. Hillblom Foundation (D.T.M.). The funders had no role in study design, data collection and analysis, decision to publish, or preparation of the manuscript.

Competing Interests: The authors have declared that no competing interests exist.

*E-mail: david.madden.tu@gmail.com

\section{Introduction}

Apoptosis is a sophisticated mechanism for eliminating unwanted cells. The signaling pathways that regulate apoptosis vary among different cell types [1,2], suggesting that apoptotic regulatory pathways are determined by differentiation status, wherein one cell lineage responds to apoptotic cues differently than others. Little is currently known about how the common precursor from which all tissues are derived - human embryonic stem cells (hESCs) - regulate entry into apoptosis. The importance of understanding these pathways is highlighted by one of the most significant obstacles to regenerative medicine: Transplantation of desired cell types contaminated with pluripotent cells can result in the formation of teratomas - tumors, usually benign, harboring differentiated cells of all lineages. If the primary pathways that govern apoptosis were to be determined in hESCs, strategies could be devised to exploit these pathways to eliminate potential teratoma-forming cells. Additionally, since large-scale expansion of hESGs remains a challenge, optimization of growth conditions could be achieved through reducing levels of apoptosis.

The most common apoptotic pathways are intrinsic pathways mediated via the mitochondrion [3,4]. Varied cell death triggers cause mitochondrial outer-membrane permeabilization (MOMP), prompting release of cytochrome $\mathrm{c}$ from the mitochondrial intermembranous space. Cytochrome c then activates caspases that effect destruction of the cell $[3,4]$. MOMP is controlled by the BCL-2 protein family, which includes both pro-apoptotic (BAX and BAK) and pro-survival family members (BCL-2, BCL-xL,
BCL-w, Al, and MCL-1), as well as the BCL-2 homology domain 3 (BH3)-only family members (BID, BAD, BIM, BIK, BLK, PUMA, NOXA, BNIP3, and HRK) [5]. The ultimate determinant of cell survival or apoptosis is the balance of active prosurvival BCL-2 family members and pro-apoptotic BCL-2 family members [5]. Not all BCL-2 family members are expressed in every cell type, and different triggers of apoptosis both activate specific pro-apoptotic BCL-2 family members and inactivate specific pro-survival BCL-2 family members $[6,7]$.

Considering their central importance in regulating apoptosis, determining the relative expression levels of the pro-apoptotic and pro-survival members of the BCL-2 family is an essential first step in describing apoptotic pathways in hESCs. In the current studies, we have addressed the following questions: (1) What is the expression of the compendium of BCL-2 family members in hESGs? (2) How does this gene expression profile compare to that in differentiated cell types? We compared the BCL-2 family member gene expression profile in pluripotent hESG lines TE06 and BG01 with those in hESC-derived neural stem cells, seven human primary cell lines from various origins, and two cancer cell lines (Table 1). The gene expression of five pro-survival BCL-2 family members, eight BH3-only BCL-2 family members, as well as BAX and BAK, was determined by quantitative reverse transcriptase polymerase chain reaction (qPCR).

We found that, compared to differentiated cells, proliferating hESCs express significantly higher levels of pro-apoptotic BCL-2 family members, including NOXA, BIK, BIM, BMF, and PUMA. Furthermore, elevated levels of these pro-apoptotic transcripts 
Table 1. Cells used in this study.

\begin{tabular}{|c|c|c|}
\hline Abbreviation & Cell Line Description & Source \\
\hline TE06 (MEF) & hESC line TE06 co-cultured with MEFs & Xianmin Zeng ${ }^{1}$ \\
\hline TE06 (MAT) & hESC line TE06 grown on Matrigel in MEF-conditioned medium & Xianmin Zeng ${ }^{1}$ \\
\hline BG01 (MEF) & hESC line BG01 co-cultured with MEFs & $\mathrm{NSCB}^{2}$ \\
\hline BG01 (MAT) & hESC line BG01 grown on Matrigel in MEF-conditioned medium & $\mathrm{NSCB}^{2}$ \\
\hline TE06-NSCs & TE06-derived neural stem cells (NSCs) & This study \\
\hline HDF & Dermal fibroblasts $^{3}$ & Invitrogen \\
\hline HEK & Epidermal keratinocytes $^{3}$ & Invitrogen \\
\hline HEMNn-LP & Epidermal melanocytes, neonatal, low pigmentation ${ }^{3}$ & Invitrogen \\
\hline HMVEC & Microvascular endothelial cells ${ }^{3}$ & Invitrogen \\
\hline HUVEC & Umbilical vein endothelial cells ${ }^{3}$ & Invitrogen \\
\hline HPASMC & Pulmonary artery smooth muscle cells ${ }^{3}$ & Invitrogen \\
\hline HMEC & Mammary epithelial cells $s^{3}$ & Invitrogen \\
\hline MCF-7 & Human cancer cell line MCF-7 & ATCC \\
\hline HeLa & Human cervix Aden carcinoma cell line & ATCC \\
\hline
\end{tabular}

${ }^{1}$ Buck Institute for Research on Aging, Novato, CA.

${ }^{2}$ National Stem Cell Bank, WiCell Research Institute, Madison, WI.

${ }^{3}$ Human primary cells.

doi:10.1371/journal.pone.0028530.t001

were not countered by elevated levels of pro-survival BCL-2 family members in hESGs. The results of this study suggest that hESGs might utilize unique mechanisms to regulate programmed cell death - mechanisms that could either be minimized to make largescale production of hESCs more feasible or exploited to minimize the risk of teratoma formation upon transplantation of hESCderived cell types.

\section{Results}

In order to determine which BCL2 family members are expressed in pluripotent human embryonic stem cells, we compared the expression profile of BCL-2 family members between hESCs and differentiated cells (see Table 1) by qPCR.

\section{Selection of housekeeping genes}

Because we chose to study gene expression in a collection of cells of seemingly disparate phenotypes, it was important to determine carefully the most appropriate reference gene(s) for normalization of the qPCR expression data. We followed the method described by Vandesompele et al. [8], which allows systematic evaluation of a collection of reference genes for those that vary least across the cell lines being analyzed. In this method, the normalization factor is a calculated value determined by the geometric mean of the expression levels of the best-performing reference genes (see methods, and REF [8]).

We selected a total of 15 genes for the purpose of normalizing qPCR data based their frequent use to normalize expression data (B2M, UBC, TBP, ACTB, YWHAZ, SDHA, RPL13A, GAPDH, HPRT1) or based on their identification as the least variant mRNAs among a large collection of gene expression data (ATP5J, SPR14, GUSB, OAZ1, PGK1, PPIA [9]). The expression stability among the set of cells outlined above, $M$ (see materials and methods for details), was calculated for each gene from our data set. From this analysis, the least stable control genes were eliminated, and new values for $M$ could be calculated from the remaining list of genes. The best-performing pair of genes, PGK1 and PPIA, was identified after step-wise exclusion of the least stable gene (highest value of $M$ ) in each of 13 iterative rounds of expression stability calculations (Figure 1).

\section{Expression of lineage-specific markers}

Once the optimum pair of reference genes was identified, we assayed the abundance of lineage-specific markers by qPCR. Expression of the transcription factors NANOG and SOX2 was

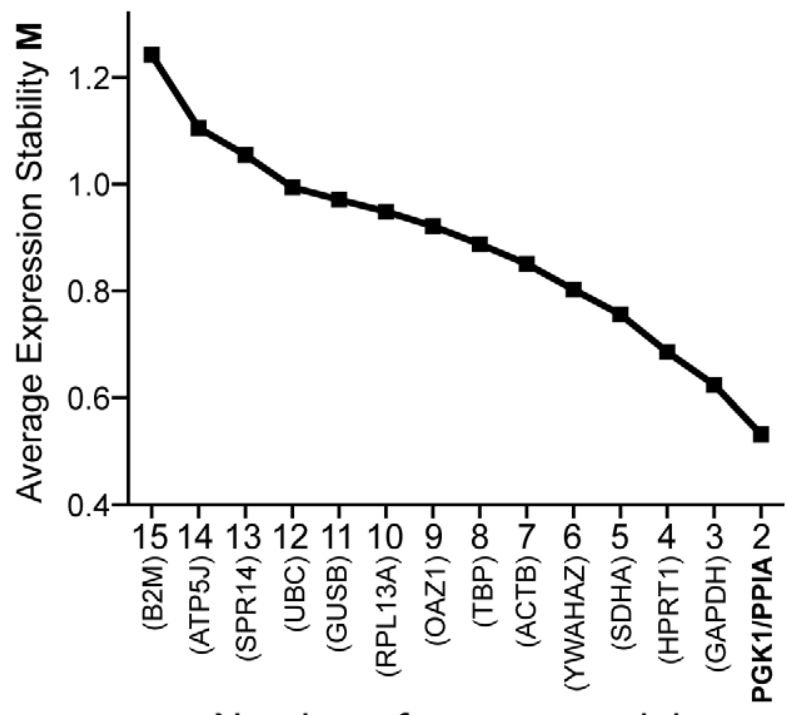

\section{Number of genes remaining}

Figure 1. Selection of optimal reference genes: PGK1 and PPIA. 15 genes were evaluated for their appropriateness for use as reference genes to normalize all subsequent data. Using a method described by Vandesompele et al. [8] genes were ranked by a stability coefficient $M$ and those genes having the lowest stability (greatest value of $M$ ) were eliminated from the subsequent round of stability calculations. The gene eliminated from each round is indicated in parentheses. doi:10.1371/journal.pone.0028530.g001 
A

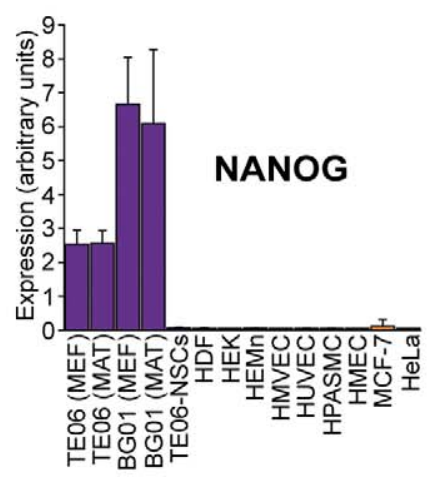

B

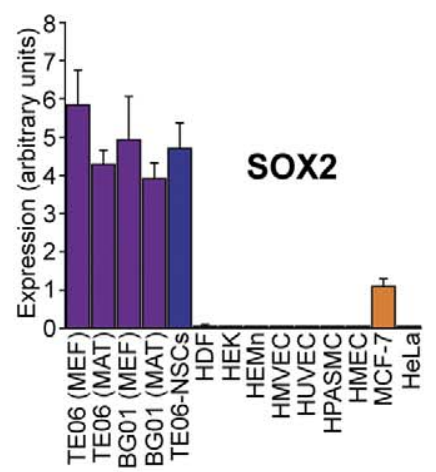

C

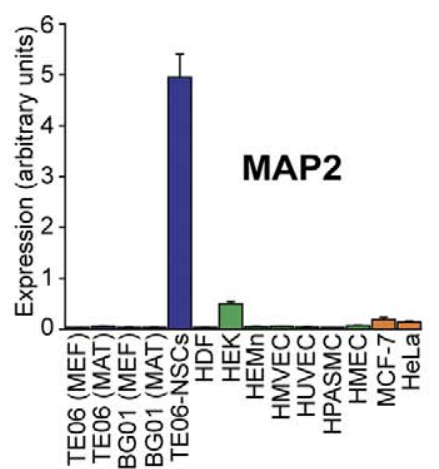

D

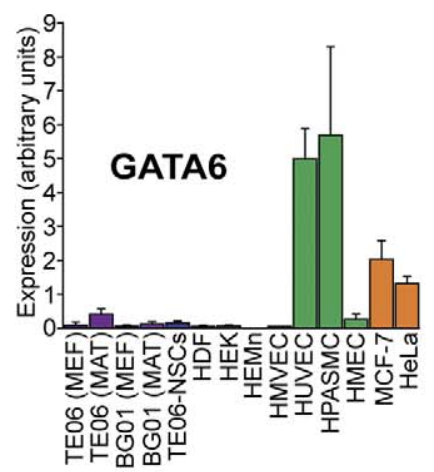

Figure 2. Cell lines express appropriate markers of pluripotency and lineage. Abundance of gene transcripts were determined by $q P C R$, and is expressed in linear arbitrary units. A. Expression of NANOG, a marker of pluripotency. B. Expression of SOX2, a marker of pluripotency and neural precursor cells. C. Expression of the microtubule-associated protein MAP2, a marker of neural cells. D. Expression of the transcription factor GATA6, a marker of heart and endoderm-derived tissues.

doi:10.1371/journal.pone.0028530.g002

primarily restricted to the hESC lines BG01 and TE06. Culturing cells with MEFs or on Matrigel in the presence of MEFconditioned medium yielded similar expression (Figure 2). Additionally, the BG01 and TE06 cells also expressed pluripotency marker proteins E-cadherin, Oct-3/4, CD9, NANOG, and PODXL (Figure 3), as well as SOX2 (data not shown). SOX2 transcript was also elevated in TE06-derived neural stem cells (TE06-NSGs), consistent with the transcription profile of NSCs $[10,11]$.

We also tested the expression status of genes normally active in differentiated cells. Elevated transcript levels of MAP2, a neuronal marker, was seen only in TE06-NSCs (Figure 2). We also found that GATA-6, a transcription factor expressed in endoderm and some mesoderm-derived tissues, was predominantly expressed in pulmonary artery smooth muscle cells (Figure 2, [12]), but was also expressed in umbilical vein endothelial cells, consistent with what has been observed previously [13,14]. Some GATA-6 expression could also be seen in the cancer lines MCF-7 and HeLa.

The immediate goal of this study was to characterize BCL-2 family members in hESCs, and determine whether their expression was either unique or absent in pluripotent human embryonic stem cells. The method we chose to identify such BCL2 members from the qPCR data was to calculate a ratio of the average BCL-2 family member expression in hESCs to the expression among the non-hES cell types. The median was used for the non-hES cell types because in many instances expression was particularly high, or low, in only one or two cell types. A simple ranking of the ratios for each BCL-2 family member revealed a surprising finding: The top five ranking BCL-2 family members having the greatest ratio of hESC:non-hESC expression were all pro-apoptotic BH3-only BCL-2 family members (Table 2). Two of the pro-apoptotic BCL-2 family members were expressed with a greater than 10-fold increase in hESGs versus non-hESGs: NOXA was expressed at an hESC:non-hESG ratio of 50, and BIK was expressed at an hESC:non-hESG ratio of 25 (Table 2). NOXA, BIK, BIM, and BMF were expressed in hESCs at levels that were significantly higher than those of the non-hES cell types (Figure 4, $p<0.001,2$-tailed student's t test, for most pair-wise comparisons between non-hES cell type and average hESC expression). Not every pro-apoptotic BCL-2 family member transcript was elevated in hESCs versus non-hES cell types, however: The pro-apoptotic BH3-only family member BNIP3 was ranked second only to the pro-survival BCL-2 family member A1 in the non-hESC:hESG expression ratio (Table 2 and Figure 5).

We expected to find instances wherein anti-apoptotic BCL-2 family members would be elevated in hESCs relative to the other cell types, so as to counter the hESC-restricted activity of the proapoptotic BH3-only BCL-2 family members NOXA, BIK, BIM, BMF, and PUMA. However, none of the five pro-survival BCL-2
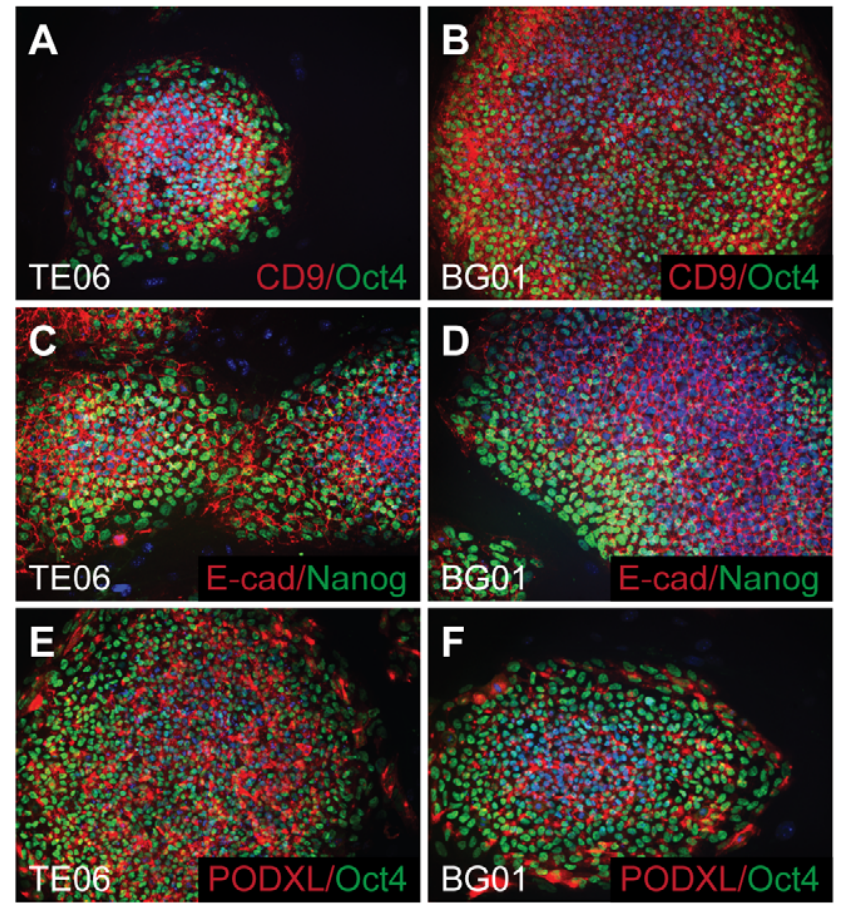

Figure 3. hESC lines BG01 and TE06 express protein markers of pluripotency. Antibodies against protein markers of pluripotency were used to label hESCs via immunofluorescence. Fixed TE06 (A, C, E) or $B G 01(B, D, F)$ cells were incubated with primary antibodies directed against CD9 (A, B), Oct4 (A, B, E, F), E-cadherin (C, D), Nanog (C, D), or PODXL $(E, F)$ followed by incubation with Alexa fluor 488 or 555 conjugated secondary antibodies. Pictures are $100 \times$ wide-field images captured on an inverted fluorescence microscope.

doi:10.1371/journal.pone.0028530.g003 
Table 2. BCL-2 family members with $\mathrm{hESC}$ or non-hESC expression bias.

\begin{tabular}{|c|c|c|c|c|}
\hline \multirow[b]{2}{*}{ Rank } & \multicolumn{2}{|c|}{ hESC $^{1}$ :Non-hESC ${ }^{2}$} & \multicolumn{2}{|c|}{ Non-hESC ${ }^{2}$ hESC $^{1}$} \\
\hline & Gene & Ratio & Gene & Ratio \\
\hline 1 & NOXA & 50.1 & $\mathrm{~A} 1$ & 102 \\
\hline 2 & BIK & 25.8 & BNIP3 & 3.3 \\
\hline 3 & $\mathrm{BIM}^{3}$ & 8.1 & $B C L-w$ & 3.1 \\
\hline 4 & BMF & 8.0 & BAD & 1.9 \\
\hline 5 & PUMA & 7.4 & BID & 1.2 \\
\hline 6 & $\mathrm{BIM}^{4}$ & 4.7 & & \\
\hline 7 & BCL2 & 3.7 & & \\
\hline \multicolumn{5}{|c|}{$\begin{array}{l}{ }^{1} \text { Mean expression of all hESC samples. } \\
{ }^{2} \text { Median of non-hESC expression values. } \\
{ }^{3} \mathrm{qPCR} \text { assay covers all three BIM transcript variants: BIM-L, BIM-EL, and BIM-S. } \\
{ }^{4} \text { Different primer/ probe set that also detects all three BIM transcripts. } \\
\text { doi:10.1371/journal.pone.0028530.t002 }\end{array}$} \\
\hline
\end{tabular}

family members we assayed demonstrated hESC-specific expression to the extent that NOXA, BIK, BMF, and PUMA did. Prosurvival BCL-2 family members were found to have either greater expression in non-hES cell types than hESCs (Al and BCL-w) or statistically insignificant differences between hESGs and nonhESCs (BCL-x and MCL-1). The one exception was the canonical anti-apoptotic molecule, BCL-2, whose average expression in hESCs was about 4-fold greater than the median non-hESC expression.

As an independent means of identifying BCL-2 family members that are uniquely expressed in either hESCs or non-hESCs, we used a clustering alignment algorithm to group the genes based on expression profiles. We included in our clustering analysis genes that are markers of pluripotency (SOX2 and NANOG) as well as markers of differentiated cells (MAP2 and GATA6). We also included the tumor suppressor p53 in our clustering analysis based on our finding that of the top five ranked genes having the greatest hESC-specific expression pattern (Table 2), three of these are direct targets of p53 transactivation activity: NOXA [15], BIK [16], and PUMA [17,18]. BAX, another known target of p53 [19], demonstrated slightly less hESC-specific expression, ranking seventh on a list of all genes from this study. Furthermore, recent evidence suggests that BIM is an indirect target or p53 [20].

We found that p53-regulated pro-apoptotic BCL-2 family members were clustered into two groups having very similar expression profiles: One group included p53 itself, along with the pluripotency marker SOX2 and the pro-apoptotic BCL-2 family members PUMA and BAX (Figure 6, correlation coefficient 0.75). The other group consisted of the pluripotency marker NANOG as well as the pro-apoptotic BCL-2 family members BIK, NOXA, and BIM (Figure 6, correlation coefficient 0.65). Although other smaller clusters of gene expression profiles were observed, none were correlated to the same degree as those seen clustered with SOX2 and NANOG. These results, taken together with the BCL2 comparison data, suggested that high basal transcript expression of potent pro-apoptotic BCL-2 family members PUMA, NOXA, $\mathrm{BIM}, \mathrm{BIK}$, and BAX, is a genuine general feature of pluripotent human embryonic stem cells.

\section{Discussion}

In this study we show that, when compared to differentiated cells or cancer cells, hESCs express elevated levels of multiple pro- apoptotic BCL-2 family members. We took advantage of a microfluidics-based qPCR platform to measure all of the human BCL-2 family members across 48 samples simultaneously. The samples consisted of 12 cell lines each having 3 or, in some cases, 4 replicate cultures, which were established from separately thawed cryologically-preserved cell stocks. Additionally, hESC lines (BG01 and TE06) were each grown under both MEF feeder and feederfree conditions. Owing to the disparate cell types evaluated for gene expression, we first measured expression levels of 15 reference genes, and identified two genes (PGK1/PPIA) that best reflected total mRNA levels. As a result of this experimental design, we are confident that the gene expression levels calculated from our qPCR experiments accurately reflect differences in abundance of mRNA transcripts among the dozen cell types studied. We hypothesized that one, or perhaps two BH3-only BCL-2 family members would be dominantly expressed in hESCs, and that these pro-apoptotic transcripts would be balanced by select pro-survival BCL-2 family members. From these patterns of expression, we hypothesized that the principal pathways governing apoptosis in hESCs could be revealed.

Contrary to our expectations, we found that hESCs express the pro-apoptotic BH3-only BCL-2 family members NOXA, BIK, BIM, BMF, and PUMA at levels far greater than that seen in the seven human primary cells, hESC-derived neural stem cells, or cancer lines (Figure 4). However, only one of the pro-survival BCL-2 family members, BCL-2, was found to be moderately elevated in hESGs compared to that seen in other cell types. Transcript abundance of two other pro-survival BCL-2 family members, BCL-x and MCL-1, was merely comparable to that seen in the other ten cell lines, and expression levels of BCL-w, and $\mathrm{Al}$ were minimal in hESGs (our assays did not distinguish between transcripts for BCL-xL and BCL-xS). What is more, unsupervised hierarchical clustering of these gene expression profiles resulted in the clustering of NOXA, BIK, BIM, BMF, and PUMA together with hESC pluripotency markers like NANOG and SOX2. We interpreted these findings as further indication that elevated expression of these BH3-only BCL-2 family members is a genuine feature of hESCs when they are either co-cultured with MEFs or grown on Matrigel with conditioned media.

Despite the fact that well over a decade has passed since the first human embryonic stem cell lines were established, we have yet to uncover the complete list of MEF-derived molecular determinants of hESC pluripotency and proliferation $[21,22]$. It is formally possible that a deficient trophic environment explains the ubiquitous observation that hESCs have elevated rates of apoptosis [23-25]. From this, it would logically follow that death of hESCs in culture might require certain pro-apoptotic BH3-only BCL-2 family members. However, it was only recently reported that BCL-2 family members play a role in governing basal rates of apoptosis in hESCs [23,26]. In one study by Ardehali et al., BCL-2 overexpression in hESCs was found to improve overall growth rates, eliminate reliance on knock-out serum replacement, increase clonogenicity after single cell dissociation, and reduce apoptosis associated with newly formed embryoid bodies [26]. In another study by Ohgushi et al., apoptosis in hESCs resulting from single cell dissociation was shown to be inhibited by overexpression of the pro-survival BCL-2 family member BCL-xL [23]. That a prosurvival BCL-2 family member could reduce dissociation-induced apoptosis and increase the overall growth rate of hESCs suggests that basal rates of apoptosis are most likely mediated by constitutive expression of pro-apoptotic $\mathrm{BH} 3$-only family members. Our data suggest that the pro-apoptotic BH3-only family members responsible for basal rates of apoptosis include NOXA, BIK, BIM, BMF, and PUMA, or a subset thereof. 
A

B

C

D

$\mathbf{E}$

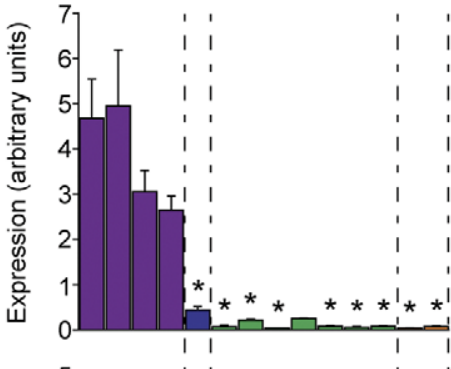

NOXA

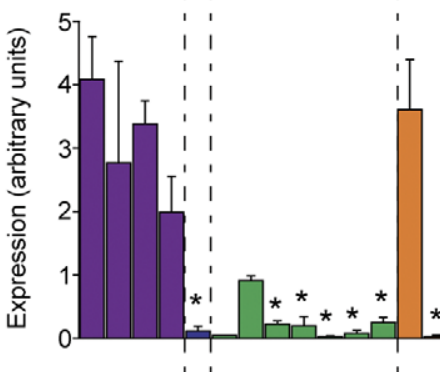

BIK

$\mathrm{BIM}^{1}$

BMF

PUMA
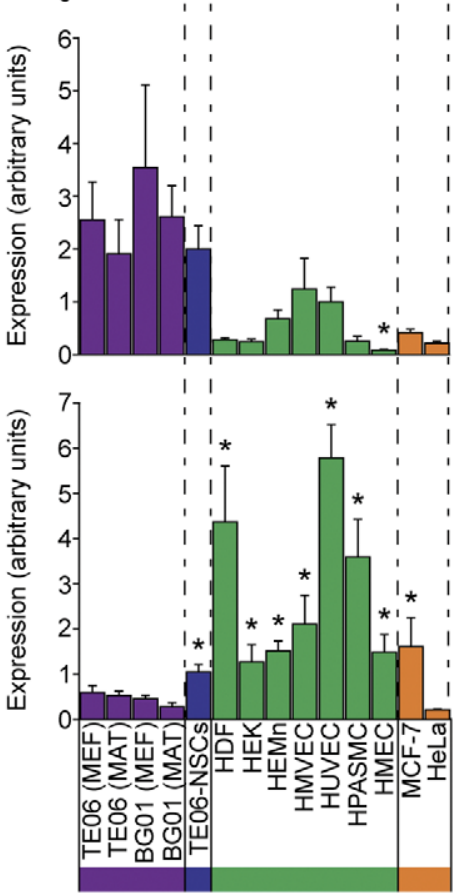

Figure 4. A cohort of pro-apoptotic BH3-only BCL-2 family members are expressed primarily in hESCs. Abundance of gene transcripts were determined by $\mathrm{QPCR}$, and is expressed in arbitrary units. A. NOXA, B. BIK, C. BIM (assay detected all three transcript variants: BIM-L, BIM-EL, and BIM-S), D. BMF, E. PUMA, F. BNIP3. Asterisks indicate values that differ significantly from the average $h E S C$ expression level ( $p<0.001$, two-tailed student's $t$ test). doi:10.1371/journal.pone.0028530.g004

Despite the constitutive overexpression of the pro-apoptotic BCL-2 family members NOXA, BIK, BIM, BMF and PUMA in hESCs, it is conceivable that the activities of these molecules would be inhibited via post-translational modifications, and that apoptosis in hESGs may be controlled by other novel signaling pathways. Accumulating evidence in the literature indicates that many BH3-only BCL-2 family members are regulated by posttranslational modifications [5]. Whereas nothing is currently known about what, if any, post-translational modifications of pro-apoptotic BH3-only BCL-2 family members might occur in hESCs, limiting the apoptotic activity of these molecules to some extent would be essential for net proliferation to occur.

For many of the pro-apoptotic BCL-2 family members, including BAD, BIM, BMF, BIK and NOXA, apoptosispromoting activity can be regulated by phosphorylation. Perhaps the most well known example of this is in the case of BAD, wherein phosphorlyation of serine residues by multiple kinases, including AKT, can promote complex formation with 14-3-3, resulting in cytosolic sequestration [5,27-39]. Similarly, ERK1/2 or RSK1/2mediated phosphorylation of BIM blunts the pro-apoptotic activity of this molecule $[5,40,41]$. Instead of being sequestered in the cytosol by 14-3-3, as in the case of BAD, BMF associates with DLC2, thereby limiting BMF's pro-apoptotic activity [5,42]. BIK is also under post-translational control, but in this case, casein kinase-mediated phosphorylation of BIK stimulates its apoptotic function $[5,43,44]$. More recently, NOXA was also discovered to be the target of phosphorylation [45]. The atypical cyclindependent kinase CDK5 phosphorylates NOXA when cells experience glucose replete conditions. Phosphorylation of NOXA causes cytosolic sequestration via an unknown mechanism and blunted apoptotic function, in addition to inducing a novel NOXA-mediated stimulation of the pentose phosphate pathway [45]. To date, no evidence of post-translational modifications of PUMA have been identified, but based on the growing list of posttranslational modifications that regulate BCL-2 family member function, post-translational modifications of PUMA remain a distinct possibility.

The picture that emerges is one in which hESCs may express pro-apoptotic BH3-only BCL-2 family members in a constitutive fashion, but that the apoptotic function of these molecules could be held in check either by complex formation with pro-survival BCL2 family members (likely BCL-xL or MCL-1) or by apoptoticinhibitory phosphorylation of NOXA, BIK, BIM, BMF and possibly PUMA, or complex formation with other inhibitory molecules (e.g., 14-3-3 for BAD, DLC2 for BMF). The advantage of such a scenario might be that apoptotic responses could be engaged more rapidly and robustly, requiring only kinase cascades to control activation of apoptosis. The danger to the cell, and a possible explanation of the challenge of growing hESCs, is that seemingly minor changes in the environment could potentially result in cell death.

In conclusion, we have shown that, compared with differentiated cells, hESCs express an unusual repertoire of BCL-2 family members. hESCs cultured under standard growth conditions contain elevated levels of transcripts encoding pro-apoptotic $\mathrm{BH} 3-$ only BCL-2 family members NOXA, BIK, BIM, BMF, and 
A

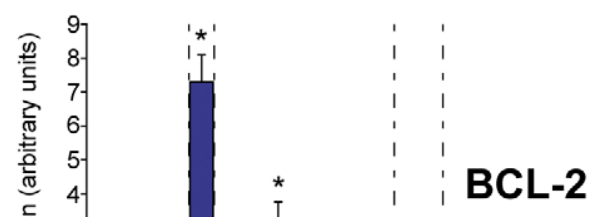

B

C

D

$\mathbf{E}$

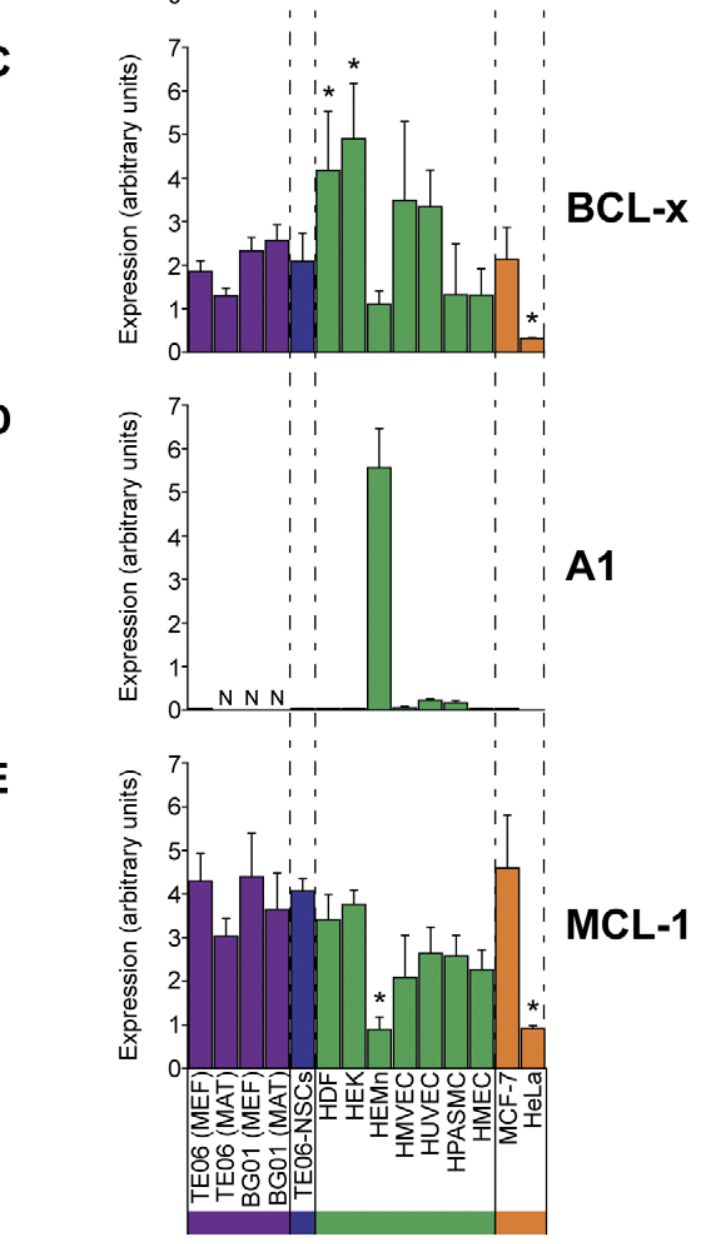

Figure 5. Expression of pro-survival BCL-2 family members. Abundance of gene transcripts were determined by $\mathrm{QPCR}$, and is expressed in linear arbitrary units. A. BCL-2, B. BCL-w, C. BCL-x (assay

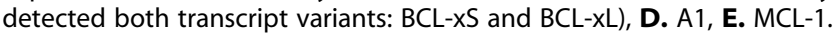
Asterisks indicate values that differ significantly from the average hESC expression level ( $p<0.001$, two-tailed student's $t$ test). doi:10.1371/journal.pone.0028530.g005

PUMA, when compared to differentiated cells. This, in light of the fact that complementary increases in pro-survival BCL-2 family members are not seen in hESCs, suggests that this expression
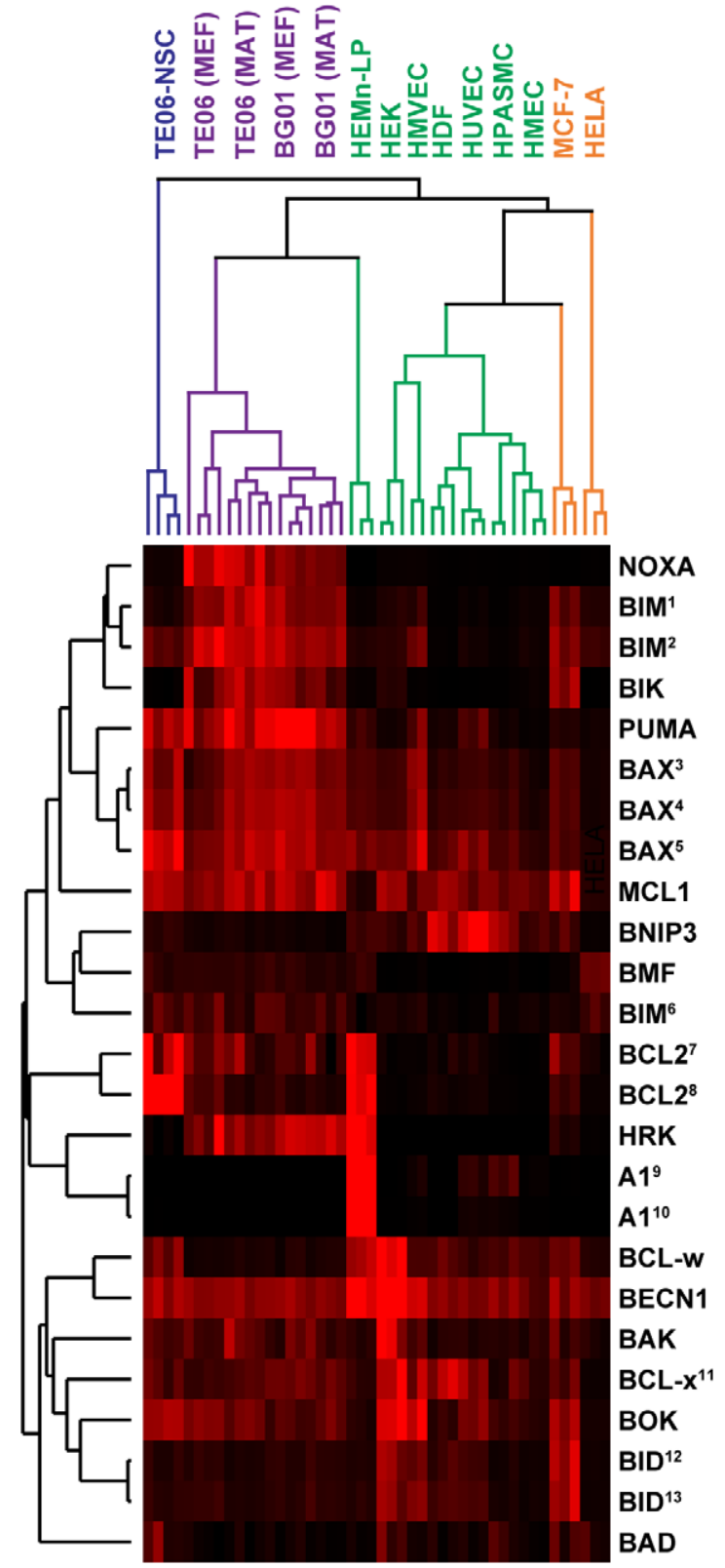

Figure 6. Hierarchical cluster analysis of BCL-2 family members in hESCs and non-hESCs. qPCR data was clustered via a two-way unsupervised clustering algorithm (Cluster 3.0) and visualized as a heat map (Java TreeView 1.1). Parallel cultures of each cell line (number): TE06-NSCs (4), TE06 MAT (4), TE06 MEF (4), BG01 MEF (4), BG01 MAT (4), HEMn-LP (3), HEK (3), HMVEC (2), HDF (3), HUVEC (3), HPASMC (3), HMEC (3), MCF-7 (3), and HeLa (3). For many genes, assays detected all or a subset of the transcript variants. ${ }^{1}$ Variants $1-7 .{ }^{2}$ Variants alpha, beta, delta, and sigma. ${ }^{3}$ Variants alpha and delta. ${ }^{4}$ Varient sigma. ${ }^{5} \mathrm{BIM}-\mathrm{L}$, BIMEL, and BIM-S. ${ }^{6}$ BIM-L, BIM-S. ${ }^{7}$ BIM-S. ${ }^{8}$ Variant $5{ }^{9}$ Variant alpha. ${ }^{10}$ Variants alpha and beta. ${ }^{11}$ Variant 1. ${ }^{12}$ Variant $2 .{ }^{13} \mathrm{BCL}-\mathrm{xL}$ and $\mathrm{BCL}-\mathrm{xS} .{ }^{14}$ Variant 3. ${ }^{15}$ Variants 1 and 2.

doi:10.1371/journal.pone.0028530.g006

profile could help to explain the elevated rates of apoptosis seen in hESCs. Recent reports demonstrating that overexpression of BCL2 [26] or BCL-xL [23] limits dissociation-induced apoptosis and increases overall growth rates is compatible with our finding that hESCs express elevated levels of pro-apoptotic BH3-only BCL-2 family members, and thus may be "primed" for apoptosis in a way 
that differentiated cells are not, and supports the notion that improvements in hESG culture methods could reduce the basal rates of apoptosis. With the large-scale propagation of hESCis remaining a major hurdle to the development of hESC-based therapies, our ability to limit the basal rate of apoptosis will be an important step toward achieving this goal.

Furthermore, given that, even in the face of elevated expression of pro-apoptotic BH3-only BCL-2 family members, hESCs are able to proliferate under standard growth conditions, hESCs might utilize novel regulatory mechanisms to control initiation of apoptosis. The importance of such a possibility is highlighted by the fact that one of the greatest obstacles to overcome in regenerative medicine is the potential of introducing teratomaforming cells during transplantation of cells with desired phenotypes. Therefore, our ability to exploit apoptotic pathways unique to hESGs could improve the clinical outcome of therapies relying on hESC-derived cells.

\section{Materials and Methods}

\section{Ethics statement}

All protocols involving hESGs were approved by the UC Davis Stem Cell Oversight Committee. The two human embryonic stem cell lines used in this study were TE06 and BG01. TE06 cells were received from the laboratory of Xianmin Zeng, Buck Institute for Research on Aging, Novato, CA. BG01 cells were purchased from the National Stem Cell Bank, WiCell Research Institute, Madison, WI.

\section{Growth of non-hESCs}

HeLa cervical carcinoma cells and MCF-7 breast carcinoma cells were purchased from the American Type Culture Collection (ATCG, Manassas, VA), and were cultured in DMEM, $2 \mathrm{mM} \mathrm{L-}$ glutamine, 50 units $/ \mathrm{ml}$ penicillin $\mathrm{G}, 50 \mathrm{ug} / \mathrm{ml}$ streptomycin sulfate (Life Technologies, Carlsbad, CA) and 10\% FBS (Sigma, St. Louis, MO). The seven human primary cell lines used in this study were purchased and grown exactly as recommended by the manufacturer using specialty media all supplied by the manufacturer (Life Technologies). Human umbilical vein endothelial cells (HUVEC, Life Technologies) were grown in LSGS-supplemented Medium 200. Human epidermal melanocytes-neonatal lightly pigmented cells (HEMn-LP, Life Technologies) were grown in HMGS-supplemented Medium 254. Human mammary microvasculature endothelial cells, neonatal dermis (HMVECnd, Life Technologies) were grown in MVGS-supplemented Medium 131. Human pulmonary artery smooth muscle cells (HPASMC, Life Technologies) were grown in SMGS-supplemented Medium 231. Human epidermal keratinocytes, neonatal (HEKn, Life Technologies) were grown in HKGS-supplemented Epilife media. Human dermal fibroblasts, neonatal (HDFn, Life Technologies) were grown in LSGS-supplemented Medium 106. Human mammary epithelial cells (HMEC, Life Technologies) were grown in HuMEC- and bovine pituitary extract-supplemented HuMEC Basal Serum Free Medium.

\section{Growth of hESCs on Mouse Embryonic Fibroblasts}

hESC lines BG01 and TE06 were propagated on mitomycin-Cinactivated MEFs (Millipore, Billerica, MA) in hESG medium: DMEM/F12 (1:1) with 20\% knockout serum replacement, $2 \mathrm{mM}$ nonessential amino acids, $2 \mathrm{mM}$ L-glutamine, 50 units $/ \mathrm{ml}$ penicillin $\mathrm{G}, 50 \mathrm{ug} / \mathrm{ml}$ streptomycin sulfate (all from Life Technologies), $0.1 \mathrm{mM}$ beta-mercaptoethanol (Millipore, Billerica, MA), and $4 \mathrm{ng} / \mathrm{ml}$ bFGF (PreproTech, Rocky Hill, NJ). hESCs were passaged enzymatically every 7 days. Briefly, cells were washed with DMEM/F12 (1:1), then incubated with collagenase IV (1 mg/ml in DMEM/F12, Life Technologies) for 40 minutes at $37^{\circ} \mathrm{C}$ and $5 \% \mathrm{CO}_{2}$. Any semi-adhered hESC colonies were pushed off the plate with a plastic pipette, and colonies were sedimented via centrifugation. Cell colonies were broken up into smaller cell aggregates by trituration of the harvested colonies in hESC medium. Triturated cells were then plated on new mitomycin-C-inactivated MEFs and incubated at $37^{\circ} \mathrm{C}$ with $5 \% \mathrm{CO}_{2}$ for $48 \mathrm{~h}$, and media was changed daily.

\section{Growth of hESCs without feeders}

MEF conditioned medium (CM) was prepared by culturing mitomycin-C-inactivated MEFs in hESC medium. After $24 \mathrm{~h}$ of incubation, the medium was collected and stored at $4^{\circ} \mathrm{C}$. The conditioned medium collected for six consecutive days from the same MEF culture was pooled, filtered through a $0.2 \mathrm{um}$ membrane (Nalgene, Rochester, NY), and aliquots were stored at $-20^{\circ} \mathrm{C}$. Before use, thawed $\mathrm{CM}$ aliquots were supplemented with an additional $4 \mathrm{ng} / \mathrm{ml} \mathrm{bFGF}$. hESCs were harvested using collagenase as described above, with the exception that colonies were triturated in CM. The dissociated hESGs were seeded on Matrigel-coated plates (1:50 dilution, BD, Franklin Lakes, NJ) in CM, and media was changed daily with CM until the hESCs reached approximately $65 \%$ confluency. Cells were passaged every 7 days.

\section{Immunocytochemistry}

Immunostaining was performed as described in Madden et al. [46]. Briefly, hESCs were co-cultured with MEFs on glass coverslips coated with gelatin. Cells were fixed in 4\% PFA for $10 \mathrm{~min}$, permeabilized in $0.05 \%$ saponin for $5 \mathrm{~min}$, and incubated in ice-cold acetone for $15 \mathrm{~min}$. Primary antibodies (all from R\&D Systems, Minneapolis, MN) were diluted with $1 \% \mathrm{wt} / \mathrm{v}$ BSA (Sigma) in PBS (PBA), and incubated with cells for $1.5 \mathrm{~h}$ at room temperature; anti-NANOG (R\&D Systems cat no. AF1997, 1:100), anti-PODXL (cat no. MAB1658, 1:100), anti-SOX2 (cat no. MAB2018, 1:100), anti-OCT3/4 (cat no. AF1759, 1:100), anti-CD9 (cat no. MAB1880, 1:100), and anti-E-Cadherin (cat no. MAB1838, 1:10). Anti-mouse or anti-rabbit secondary antibodies conjugated to either Alexa Fluor 488 or Alexa Fluor 555 (Life Technologies) were diluted 1:200 in PBA and incubated for $1.5 \mathrm{~h}$ at room temperature in the dark. Washed cells were then post-fixed with $2 \mathrm{ug} / \mathrm{ml}$ Hoechst 33342 (Life Technologies) in 4\% PFA for $10 \mathrm{~min}$ and mounted on to glass slides with SlowFade antifade reagent following the manufacturer's instructions (Life Technologies). Fluorescence images were viewed with Nikon fluorescence objectives using a Nikon TE300 (Nikon Inc., Melville, NY). Images were captured with a Photometrics CoolSNAP EZ digital camera (Roper Scientific, Inc., Germany) using Simple PCI software (Compix Inc. Imaging Systems, Cranberry Township, PA). The brightness, contrast, and unsharp mask functions of Adobe Photoshop CS were used to optimize the images (Adobe Systems Inc., San Jose California).

\section{Karyotypic analysis}

Karyotypic analysis of our expanded banks of hESCis (BG01 and TE06) was performed by Cell Line Genetics (Madison, WI). See Figure S1.

\section{RNA Isolation}

For the purposes of this study, cultures were established from liquid nitrogen storage from three or, in some cases, four vials of a previously expanded cell line. Replicate cultures were maintained 
in parallel until sufficient quantities for RNA extraction were grown. Independently established cultures were never mixed or combined with other cultures of the same line.

RNA was isolated from cells grown in parallel cultures (replicate n): BG01 on MEFs (4), BG01 on Matrigel (4), TE06 on MEFs (4), TE06 on Matrigel (4), TE06-derived NSCs (4), human pulmonary smooth muscle cells (3), human mammary epithelial cells (3), human epidermal keratinocytes (3), human epidermal fibroblasts (3), human umbilical vein endothelial cells (3), human microvascular endothelial cells (3), human epidermal melanocytes (3), the cancer lines HeLa (3) and MCF7 (3). RNA was also isolated from mouse embryonic fibroblasts to verify that qPCR primer-assay pairs were human-specific.

hESC colonies were harvested in a similar fashion used for routine passaging with the exception that cells were treated with collagenase for $20 \mathrm{~min}$ only. Colonies were collected by centrifugation $(200 \times \mathrm{g})$ for $2 \mathrm{~min}$, the supernatant was aspirated, cells were frozen in a bath of dry-ice/ ethanol, and transferred to a $-80^{\circ} \mathrm{C}$ cryo-freezer for storage. The seven primary cell lines (human pulmonary smooth muscle cells, human mammary epithelial cells, human epidermal keratinocytes, human epidermal fibroblasts, human umbilical vein endothelial cells, human microvascular endothelial cells, human epidermal melanocytes) as well as the two cancer lines (HeLa and MCF-7) were harvested by trypsinization, neutralized with trypsin inhibitor (primary cells only), collected by centrifugation, decanted, and frozen on dryice/ ethanol. Frozen pellets were transferred to a $-80^{\circ} \mathrm{C}$ cryofreezer for storage. After eight months of storage, RNA was isolated using Quiagen RNeasy kits (Quiagen, Valencia, CA).

\section{Microfluidic Polymerase Chain Reaction}

We used a BioMark ${ }^{\mathrm{TM}}$ real-time PCR system (Fluidigm, San Francisco, CA, USA) in conjunction with a BioMark 48.48 Dynamic Array, which has high throughput capacity due to its integrated fluidic circuit feature [47]. First-strand cDNA was prepared using SuperScript III First-Strand Synthesis SuperMix for qPCR according to the manufacturer's recommendations (Life Technologies). Prior to analysis, cDNA from each sample was preamplified using a $0.2 \times$ mixture of 25 Universal Probe Library gene expression assays (Roche Applied Science, Indianapolis, IN) and TaqMan ${ }^{\circledR}$ PreAmp Master Mix (Life Technologies). cDNA $(1.25 \mu \mathrm{l})$ was amplified in a reaction $(5 \mu \mathrm{l})$ for 14 cycles according to the manufacturer's recommendations. At the end of thermal cycling, the reactions were diluted 1:5 in low EDTA TE $(10 \mathrm{mM}$ Tris-HCl, pH 8.0; $0.1 \mathrm{mM}$ EDTA, pH 8.0). Samples were prepared for loading into the dynamic array by mixing TaqMan ${ }^{\circledR}$ Universal Master Mix (2.5 $\mu$, Life Technologies), DA Sample Loading Reagent (0.25 $\mu$ l, PN 85000735; Fluidigm) and preamplified cDNA (2.25 $\mu \mathrm{l})$. The 48.48 Dynamic Array (PN BMKM-48.48; Fluidigm) was primed according to the manufacturer's recommendations. Samples $(5 \mu \mathrm{l})$ were loaded into separate sample inlets on the Dynamic Array. Gene expression assays (Roche Applied Science) prepared as $20 \times$ solutions were diluted to $10 \times$ using the DA Assay Loading Reagent (PN 85000735; Fluidigm) and aliquots $(5 \mu \mathrm{l})$ were loaded into separate reagent inlets on the Dynamic Array. Each assay was run as technical triplicates or quadruplicates, and loading positions across the array were randomized. The dynamic array was placed on a Nanoflex ${ }^{\mathrm{TM}}$ 4-IFC Controller (Fluidigm) for loading and mixing. After loading, the dynamic array was placed on the BioMark ${ }^{\text {TM }}$ real-time PCR system for thermal cycling and real-time imaging of the reactions. Thermal cycling conditions were: $50^{\circ} \mathrm{C}$ for $2 \mathrm{~min}$ with AmpErase UNG (Life Technologies), $95^{\circ} \mathrm{C}$ for $10 \mathrm{~min}$ hot start, and 40 cycles of $95^{\circ} \mathrm{C}$ for $15 \mathrm{~s}$ and $60^{\circ} \mathrm{C}$ for $1 \mathrm{~min}$. Ct values were determined using BioMark real-time PCR analysis software. All other calculations, including reference gene stability, were performed using Microsoft Excel (Microsoft, Redmond, WA).

We used the Universal Probe Library (Roche Applied Science, Indianapolis, IN) set of sequence-specific probes and relied on the Universal Probe Library assay design tool (www.roche-appliedscience.com) to choose primer sequences and the appropriate fluorescent sequence-specific probe. All primers were purchased from Sigma (St Louis, MO). For the list of all primer and probe sequences used in this study, see Table S1.

\section{Data processing}

$\mathrm{C}_{\mathrm{t}}$ values were determined from the 47 samples based upon the mean of the $\mathrm{C}_{\mathrm{t}} \mathrm{s}$ from technical replicates of assays used to detect 15 different putative reference genes. Next, the set of $47 \mathrm{C}_{t}$ values was converted to relative quantity for each of the 15 gene assays. Calculations were performed as described by Vandesompele et al. [8]. Briefly, a new array $A_{j k}$ was calculated as the $\log _{2}$-transformed expression ratios $a_{i j} / a_{i k}$ for each combination of two control genes $j$ and $k$ ( $i=1$ to $m$ elements, $m=47$ for this study, equation 1$)$. The pairwise variation $V_{j k}$ was calculated as the standard deviation of the $A_{j k}$ elements (equation 2). The stability of the control gene $j\left(M_{j}\right)$ was defined as the arithmetic mean of all pairwise variations $V_{j k}$ (equation 3).

$$
\begin{gathered}
(\forall j, k \in[1, n] \text { and } j \neq k): \\
A_{j k}=\left\{\log _{2}\left(\frac{a_{1 j}}{a_{1 k}}\right), \log _{2}\left(\frac{a_{2 j}}{a_{2 k}}\right), \ldots, \log _{2}\left(\frac{a_{m j}}{a_{m k}}\right)\right\} \\
=\left\{\log _{2}\left(\frac{a_{i j}}{a_{i k}}\right)\right\}_{i=1 \rightarrow m} \\
V_{j k}=\operatorname{st} \cdot \operatorname{dev}\left(A_{j k}\right) \\
M_{j}=\frac{\sum_{k=1}^{n} V_{j k}}{n-1}
\end{gathered}
$$

The 15 reference genes were ranked in order of their stability $M$ : The reference gene having the lowest gene stability (highest value of $M$, and in this case B2M) was eliminated from a new data set having 14 control genes, and new values of $A_{j k}, V_{j k}$, and $M_{j}$ were calculated for the new data set. This process was repeated iteratively until only two genes were remaining of the initial 15: PPIA and PGK1. The geometric mean of these two genes was used to normalize the expression of all other genes for each of the subsequent six dynamic arrays where expression levels of query genes were assayed.

\section{Clustering Analysis}

Gene expression data were analyzed using the clustering analysis software Cluster (Stanford) based on a clustering algorithm described previously [48]. The similarity metric was absolute correlation (centered) using the average linkage clustering method. The clustered data was displayed as a heat map using Java TreeView [49].

\section{Supporting Information}

Figure S1 Karyotypic analysis of hESG lines BG01 and TE06.

(TIF) 
Table S1 qPGR primer and UPL probe sequences used in this study.

(XLS)

\section{Acknowledgments}

The authors would like to thank Krysta Felkey in the genomics core of the Buck Institute for Research on Aging for running the arrays, as well as the contributions made by Alejandra Davila and Tim Roth in helping to design primer sequences. We thank Tobias Wittkop and Sean Mooney for

\section{References}

1. Yuan J, Kroemer G (2010) Alternative cell death mechanisms in development and beyond. Genes Dev 24: 2592-2602. doi:10.1101/gad.1984410.

2. Conradt B (2009) Genetic control of programmed cell death during animal development. Annu Rev Genet 43: 493-523. doi:10.1146/annurev.genet. 42.110807.091533.

3. Tait SWG, Green DR (2010) Mitochondria and cell death: outer membrane permeabilization and beyond. Nat Rev Mol Cell Biol 11: 621-632. doi:10.1038/ nrm2952.

4. Bredesen DE, Rao RV, Mehlen P (2006) Cell death in the nervous system. Nature 443: 796-802. doi:10.1038/nature05293.

5. Chipuk JE, Moldoveanu T, Llambi F, Parsons MJ, Green DR (2010) The BCL2 Family Reunion. Mol Cell 37: 299-310. doi:10.1016/j.molcel.2010.01.025.

6. Lopez H, Zhang L, George NM, Liu X, Pang X, et al. (2010) Perturbation of the Bcl-2 network and an induced Noxa/Bcl-xL interaction trigger mitochondrial dysfunction after DNA damage. J Biol Chem 285: 15016-15026. doi:10.1074/ jbc.M109.086231.

7. Zhang L, Lopez H, George NM, Liu X, Pang X, et al. (2011) Selective involvement of BH3-only proteins and differential targets of Noxa in diverse apoptotic pathways. Cell Death Differ 18: 864-873. doi:10.1038/cdd.2010.152.

8. Vandesompele J, De Preter K, Pattyn F, Poppe B, Van Roy N, et al. (2002) Accurate normalization of real-time quantitative RT-PCR data by geometric averaging of multiple internal control genes. Genome Biol 3: RESEARCH0034.

9. de Jonge HJM, Fehrmann RSN, de Bont ESJM, Hofstra RMW, Gerbens F, et al. (2007) Evidence based selection of housekeeping genes. PLoS ONE 2: e898. doi:10.1371/journal.pone.0000898.

10. Rex M, Orme A, Uwanogho D, Tointon K, Wigmore PM, et al. (1997) Dynamic expression of chicken Sox 2 and Sox 3 genes in ectoderm induced to form neural tissue. Dev Dyn 209: 323-332. doi:10.1002/(SICI)10970177(199707)209:3<323::AID-AJA7>3.0.CO;2-K.

11. Cimadamore F, Fishwick K, Giusto E, Gnedeva K, Cattarossi G, et al. (2011) Human ESG-Derived Neural Crest Model Reveals a Key Role for SOX2 in Sensory Neurogenesis. Cell Stem Cell 8: 538-551. doi:10.1016/j.stem. 2011.03.011.

12. Suzuki E, Evans T, Lowry J, Truong L, Bell DW, et al. (1996) The human GATA-6 gene: structure, chromosomal location, and regulation of expression by tissue-specific and mitogen-responsive signals. Genomics 38: 283-290. doi:10.1006/geno.1996.0630.

13. Umetani M, Mataki C, Minegishi N, Yamamoto M, Hamakubo T, et al. (2001) Function of GATA transcription factors in induction of endothelial vascular cell adhesion molecule-1 by tumor necrosis factor-alpha. Arterioscler Thromb Vasc Biol 21: 917-922.

14. Peng Y, Jahroudi N (2003) The NFY transcription factor inhibits von Willebrand factor promoter activation in non-endothelial cells through recruitment of histone deacetylases. J Biol Chem 278: 8385-8394. doi:10.1074/jbc.M213156200.

15. Oda E, Ohki R, Murasawa H, Nemoto J, Shibue T, et al. (2000) Noxa, a BH3only member of the Bcl-2 family and candidate mediator of p53-induced apoptosis. Science 288: 1053-1058.

16. Mathai JP, Germain M, Marcellus RC, Shore GC (2002) Induction and endoplasmic reticulum location of BIK/NBK in response to apoptotic signaling by E1A and p53. Oncogene 21: 2534-2544. doi:10.1038/sj.onc.1205340.

17. Nakano K, Vousden KH (2001) PUMA, a novel proapoptotic gene, is induced by p53. Mol Cell 7: 683-694

18. Yu J, Zhang L, Hwang PM, Kinzler KW, Vogelstein B (2001) PUMA induces the rapid apoptosis of colorectal cancer cells. Mol Cell 7: 673-682.

19. Miyashita T, Reed JC (1995) Tumor suppressor p53 is a direct transcriptional activator of the human bax gene. Cell 80: 293-299.

20. Happo L, Cragg MS, Phipson B, Haga JM, Jansen ES, et al. (2010) Maximal killing of lymphoma cells by DNA damage-inducing therapy requires not only the p53 targets Puma and Noxa, but also Bim. Blood 116: 5256-5267. doi:10.1182/blood-2010-04-280818.

21. Hasegawa K, Pomeroy JE, Pera MF (2010) Current technology for the derivation of pluripotent stem cell lines from human embryos. Cell Stem Cell 6: 521-531. doi:10.1016/j.stem.2010.05.010.

22. Thomson JA, Itskovitz-Eldor J, Shapiro SS, Waknitz MA, Swiergiel JJ, et al. (1998) Embryonic stem cell lines derived from human blastocysts. Science 282: 1145-1147.

23. Ohgushi M, Matsumura M, Eiraku M, Murakami K, Aramaki T, et al. (2010) Molecular pathway and cell state responsible for dissociation-induced apoptosis help with clustering analysis, and Shona Mookerjee for critical improvement of the manuscript.

\section{Author Contributions}

Conceived and designed the experiments: DTM SM DEB. Performed the experiments: DD-K DTM. Analyzed the data: DTM SM. Contributed reagents/materials/analysis tools: DTM SM DEB. Wrote the paper: DTM.

in human pluripotent stem cells. Cell Stem Cell 7: 225-239. doi:10.1016/ j.stem.2010.06.018.

24. Pyle AD, Lock LF, Donovan PJ (2006) Neurotrophins mediate human embryonic stem cell survival. Nat Biotechnol 24: 344-350. doi:10.1038/ nbt1189.

25. Oin H, Yu T, Oing T, Liu Y, Zhao Y, et al. (2007) Regulation of apoptosis and differentiation by p53 in human embryonic stem cells. J Biol Chem 282: 5842-5852. doi:10.1074/jbc.M610464200.

26. Ardehali R, Inlay MA, Ali SR, Tang C, Drukker M, et al. (2011) Overexpression of BCL2 enhances survival of human embryonic stem cells during stress and obviates the requirement for serum factors. Proc Natl Acad Sci USA 108: 3282-3287. doi:10.1073/pnas. 1019047108.

27. Cotteret S, Jaffer ZM, Beeser A, Chernoff J (2003) p21-Activated kinase 5 (Pak5) localizes to mitochondria and inhibits apoptosis by phosphorylating BAD. Mol Cell Biol 23: 5526-5539.

28. Danial NN, Gramm CF, Scorrano L, Zhang C-Y, Krauss S, et al. (2003) BAD and glucokinase reside in a mitochondrial complex that integrates glycolysis and apoptosis. Nature 424: 952-956. doi:10.1038/nature01825.

29. Danial NN, Walensky LD, Zhang G-Y, Choi CS, Fisher JK, et al. (2008) Dual role of proapoptotic BAD in insulin secretion and beta cell survival. Nat Med 14: 144-153. doi: 10.1038/nm1717.

30. Datta SR, Dudek H, Tao X, Masters S, Fu H, et al. (1997) Akt phosphorylation of BAD couples survival signals to the cell-intrinsic death machinery. Cell 91: 231-241.

31. Datta SR, Katsov A, Hu L, Petros A, Fesik SW, et al. (2000) 14-3-3 proteins and survival kinases cooperate to inactivate $\mathrm{BAD}$ by $\mathrm{BH} 3$ domain phosphorylation. Mol Cell 6: 41-51.

32. del Peso L, González-García M, Page C, Herrera R, Nuñez G (1997) Interleukin-3-induced phosphorylation of BAD through the protein kinase Akt. Science 278: 687-689.

33. Donovan N, Becker EBE, Konishi Y, Bonni A (2002) JNK phosphorylation and activation of BAD couples the stress-activated signaling pathway to the cell death machinery. J Biol Chem 277: 40944 40949. doi:10.1074/jbc.M206113200.

34. Fox CJ, Hammerman PS, Cinalli RM, Master SR, Chodosh LA, et al. (2003) The serine/threonine kinase Pim-2 is a transcriptionally regulated apoptotic inhibitor. Genes Dev 17: 1841-1854. doi:10.1101/gad.1105003.

35. Harada H, Andersen JS, Mann M, Terada N, Korsmeyer SJ (2001) p70S6 kinase signals cell survival as well as growth, inactivating the pro-apoptotic molecule BAD. Proc Natl Acad Sci USA 98: 9666-9670. doi:10.1073/ pnas. 171301998.

36. Konishi Y, Lehtinen M, Donovan N, Bonni A (2002) Cdc2 phosphorylation of BAD links the cell cycle to the cell death machinery. Mol Cell 9: 1005-1016.

37. Schürmann A, Mooney AF, Sanders LC, Sells MA, Wang HG, et al. (2000) p21activated kinase 1 phosphorylates the death agonist bad and protects cells from apoptosis. Mol Cell Biol 20: 453-461.

38. Tan Y, Ruan H, Demeter MR, Comb MJ (1999) p90(RSK) blocks badmediated cell death via a protein kinase C-dependent pathway. J Biol Chem 274: 34859-34867.

39. Yan B, Zemskova M, Holder S, Chin V, Kraft A, et al. (2003) The PIM-2 kinase phosphorylates BAD on serine 112 and reverses BAD-induced cell death. J Biol Chem 278: 45358-45367. doi:10.1074/jbc.M307933200.

40. Dehan E, Bassermann F, Guardavaccaro D, Vasiliver-Shamis G, Cohen M, et al. (2009) betaTrCP- and Rsk1/2-mediated degradation of BimEL inhibits apoptosis. Mol Cell 33: 109-116. doi:10.1016/j.molcel.2008.12.020.

41. Ley R, Balmanno K, Hadfield K, Weston C, Cook SJ (2003) Activation of the ERK1/2 signaling pathway promotes phosphorylation and proteasomedependent degradation of the BH3-only protein, Bim. J Biol Chem 278: 18811-18816. doi:10.1074/jbc.M301010200.

42. Puthalakath H, Villunger A, O'Reilly LA, Beaumont JG, Coultas L, et al. (2001) Bmf: a proapoptotic $\mathrm{BH} 3$-only protein regulated by interaction with the myosin $\mathrm{V}$ actin motor complex, activated by anoikis. Science 293: 1829-1832. doi:10.1126/science.1062257.

43. Li YM, Wen Y, Zhou BP, Kuo H-P, Ding Q, et al. (2003) Enhancement of Bik antitumor effect by Bik mutants. Cancer Res 63: 7630-7633.

44. Verma S, Zhao LJ, Chinnadurai G (2001) Phosphorylation of the pro-apoptotic protein BIK: mapping of phosphorylation sites and effect on apoptosis. J Biol Chem 276: 4671-4676. doi:10.1074/jbc.M008983200. 
45. Lowman XH, Mcdonnell MA, Kosloske A, Odumade OA, Jenness C, et al. (2010) The Proapoptotic Function of Noxa in Human Leukemia Cells Is Regulated by the Kinase Cdk5 and by Glucose. Mol Cell 40: 823-833. doi:10.1016/j.molcel.2010.11.035.

46. Madden DT, Egger L, Bredesen DE (2007) A calpain-like protease inhibits autophagic cell death. Autophagy 3: 519-522.
47. Spurgeon SL, Jones RC, Ramakrishnan R (2008) High throughput gene expression measurement with real time PCR in a microfluidic dynamic array. PLoS ONE 3: e1662. doi:10.1371/journal.pone.0001662.

48. Eisen MB, Spellman PT, Brown PO, Botstein D (1998) Cluster analysis and display of genome-wide expression patterns. Proc Natl Acad Sci USA 95: 14863-14868.

49. Saldanha AJ (2004) Java Treeview-extensible visualization of microarray data. Bioinformatics 20: 3246-3248. doi:10.1093/bioinformatics/bth349. 